\title{
The impact of chemotherapy-induced neutropenia on the outcome of direct-to-implant immediate breast reconstruction
}

\author{
Yong Sakong^, Man Ki Choi, Jun Ho Lee \\ Department of Plastic and Reconstructive Surgery, Yeungnam University College of Medicine, Daegu, Korea \\ Contributions: (I) Conception and design: JH Lee; (II) Administrative support: JH Lee; (III) Provision of study materials or patients: Y Sakong, MK \\ Choi; (IV) Collection and assembly of data: Y Sakong, MK Choi; (V) Data analysis and interpretation: Y Sakong; (VI) Manuscript writing: All \\ authors; (VII) Final approval of manuscript: All authors. \\ Correspondence to: Jun Ho Lee. Department of Plastic and Reconstructive Surgery, Yeungnam University College of Medicine, 170 Hyeonchung-ro, \\ Nam-gu, Daegu 42415, Korea. Email: junojunho@gmail.com.
}

\begin{abstract}
Background Adjuvant chemotherapy can cause neutropenia by inhibition of hematopoiesis. However, little information is known about the effects of chemotherapy-induced neutropenia (CIN) on the outcomes of direct-to-implant (DTI) immediate breast reconstruction after skin-sparing mastectomy.

Methods: A retrospective review was performed for all patients with DTI immediate breast reconstruction after skin-sparing mastectomy (n=372) between January 2011 and December 2019. The demographic and complication of patients who experienced CIN during chemotherapy and those who did not were compared. Results: Major infection requiring surgical management occurred in 4 patients $(7.1 \%)$ in the CIN group $(\mathrm{n}=56)$ and $2(3.6 \%)$ in the non-CIN group $(\mathrm{n}=55)$. Minor infection requiring antibiotics treatment occurred in 1 patient $(1.8 \%)$ in the CIN group and $1(1.8 \%)$ in the non-CIN group. Skin necrosis occurred in 7 patients $(12.5 \%)$ in CIN group and 11 patients (19.6\%) in non-CIN group. There were no significant difference in incidence of all complications between two groups.

Conclusions: CIN may not significantly increase the incidence of severe complications in the patients who received adjuvant chemotherapy after DTI immediate breast reconstruction. However, close observation is required for possible breast complication and adequate treatment is needed.
\end{abstract}

Keywords! Breast implantation; chemotherapy; surgical wound infection

Submitted Mar 03, 2021. Accepted for publication Mar 31, 2021.

doi: 10.21037/apm-21-508

View this article at: http://dx.doi.org/10.21037/apm-21-508

\section{Introduction}

Currently, direct-to-implant (DTI) immediate breast reconstruction is a well-accepted surgical treatment for patients who need skin-sparing mastectomy in breast cancer. Adjuvant therapy for breast cancer can include chemotherapy, hormonal therapy, radiotherapy, the targeted drug trastuzumab (Herceptin ${ }^{\circledR}$ ), or a combination of treatments. Among these treatments, adjuvant chemotherapy is increasingly being used in women with breast cancer (1).
Adjuvant chemotherapy increases the survival rate and decreases the recurrence rate in early-stage breast cancer (2). Unfortunately, adjuvant chemotherapy can cause chemotherapy-induced neutropenia (CIN) by suppressing the hematopoietic system $(3,4)$. A deficit of neutrophils makes the patients vulnerable to infection and induce poor wound healing.

Even though adjuvant chemotherapy following immediate breast reconstruction was demonstrated to not increase the risk of complications $(5,6)$, no information

$\wedge$ ORCID: 0000-0003-3185-5231. 
has been published about the influences of CIN on the outcomes of DTI breast reconstruction after skin-sparing mastectomy. In breast cancer patients, the occurrence of infection after breast reconstruction using implants is a serious side effect and is of great concern to patients, may require secondary operation (7). Therefore, it is a major concern for plastic surgeons whether acute infection is induced in the breast when a patient has CIN during chemotherapy. This study aims to investigate the effect of CIN on breast implant complication in patients who underwent DTI breast reconstruction.

We present the following article in accordance with the STROBE reporting checklist (available at http://dx.doi. org/10.21037/apm-21-508).

\section{Methods}

The study was conducted in accordance with the Declaration of Helsinki (as revised in 2013). The study was approved by institutional review board of Yeungnam University Hospital (No. 2021-01-043: the registration number of ethics board) and informed consent were taken from all the patients of clinical photographs at the figure. A retrospective review was performed to analyze outcomes of patients who underwent adjuvant chemotherapy after DTI breast reconstruction procedure between January of 2011 and December of 2019 performed by the senior surgeon (JH Lee). All implants were placed in the subpectoral plane with acellular dermal matrix (ADM). ADM supported the implant at the lower margin of the pectoralis muscle. Three products of ADM; CryoDerm (CG BIO Corp., Seongnam, Korea), AlloDerm (LifeCell Corp., Branchburg, NJ, USA), and MegaDerm (L\&C Bio, Seoul, Korea) were used. Silicone textured type breast implant was used during the study. To exclude patient who had CIN before the breast reconstruction, patients who received neoadjuvant therapy were excluded. In case of patients who need postoperative radiotherapy, we used breast expanders due to complications such as soft tissue injury, ulceration, and microangiopathic changes caused by radiotherapy. Therefore, patients with breast reconstructed with expanders were also excluded. One or two drainages were inserted after breast reconstruction. It was removed when discharge was less than $20 \mathrm{cc}$ for two consecutive days. Adjuvant chemotherapy was started under agreement of plastic surgeon and breast surgeon, and chemotherapy was delayed if the wound condition was not healed well enough.
The severity of neutropenia is associated with the relative risk of infection (5). Three grades are used to classify the severity of neutropenia based on the absolute neutrophil count (ANC) and are defined as follows: mild neutropenia with an ANC of 1,000-1,500 cells/ $\mu \mathrm{L}$, moderate neutropenia with an ANC of $500-1,000$ cells $/ \mu \mathrm{L}$, and severe neutropenia with ANC of $<500$ cells $/ \mu \mathrm{L}(5,7)$. This study included patients who experienced severe CIN (ANC $<500 \mathrm{~mm}^{3}$ ) during adjuvant chemotherapy. All the patients in the CIN group were managed with multiple doses of filgrastim injection (Leucostim ${ }^{\circledR}$, Dong-A Socio Holdings Co., Korea), a human recombinant granulocyte colony-stimulating factor (G-CSF), until recovery from neutropenia. When breast infection was strongly suspected due to symptoms such as breast pain, erythema, and swelling, intravenous vancomycin and tazime were administered immediately.

Patients were divided into two groups according to the occurrence of CIN. The demographic data, comorbidities, adjuvant chemotherapy regime, postoperative day (POD) of drain removal were investigated. Examples of postoperative complications were as follows: major infection (infection leading to implant removal), minor infection (infection treated by intravenous antibiotic), capsular contracture, skin necrosis. Only complications that occurred between 1 month before and after the diagnosis of CIN were counted in CIN group.

\section{Statistical analysis}

Statistical analysis was performed using by SPSS version 19.0. Patient-related characteristics were compared among the two groups. Descriptive statistics were used to compare the postoperative complications across two groups. Pearson chi-square test was performed to analyze categorical variables and to check for correlations between CIN and complications. A value of $\mathrm{P}<0.05$ indicated statistical significance.

\section{Results}

Between January 2011 and December 2019, 372 patients underwent DTI breast reconstruction. Among them, 111 patients received adjuvant chemotherapy (Table 1). All the patients underwent unilateral reconstruction, so 111 breasts were analyzed. Fifty-six patients had CIN (54\%) during chemotherapy and 55 patients did not (46\%). The mean 
Table 1 Demographic of non-CIN group and CIN group

\begin{tabular}{|c|c|c|}
\hline Group & Non-CIN group & CIN group \\
\hline No. of patients & 55 & 56 \\
\hline Age & 46.89 & 45.42 \\
\hline Body mass index $\left(\mathrm{kg} / \mathrm{m}^{2}\right)$ & 23.57 & 22.95 \\
\hline \multicolumn{3}{|l|}{ Underlying disease } \\
\hline Hypertension & 9 & 16 \\
\hline Diabetes & 15 & 22 \\
\hline Thyroid cancer & 3 & 0 \\
\hline Dyslipidemia & 0 & 1 \\
\hline Lowest ANC (cells $/ \mu \mathrm{L})$ & - & $\begin{array}{c}268.04 \\
(10.01-956.48)\end{array}$ \\
\hline $\begin{array}{l}\text { DTI-adjuvant chemotherapy } \\
\text { term (day) }\end{array}$ & $33.04 \pm 79.05$ & $17.66 \pm 19.74$ \\
\hline \multicolumn{3}{|l|}{ ADM } \\
\hline CG CryoDerm & 10 & 23 \\
\hline MegaDerm & 33 & 21 \\
\hline AlloDerm & 12 & 12 \\
\hline \multicolumn{3}{|l|}{ Implant type } \\
\hline Textured & 55 & 56 \\
\hline Smooth & - & - \\
\hline POD of drain removal (day) & 16.79 & 15.81 \\
\hline \multicolumn{3}{|l|}{ Breast cancer stage } \\
\hline 0 & 1 & 0 \\
\hline I & 11 & 17 \\
\hline II & 40 & 34 \\
\hline III & 2 & 4 \\
\hline IV & 1 & 1 \\
\hline \multicolumn{3}{|l|}{ Adjuvant chemotherapy regime } \\
\hline 5-fluorouracil & 1 & 1 \\
\hline Doxorubicin & 1 & - \\
\hline Docetaxel & 1 & 1 \\
\hline$A C$ & 38 & 37 \\
\hline $\mathrm{TC}$ & 2 & 6 \\
\hline AT & 1 & 1 \\
\hline FC & 1 & - \\
\hline FEC & 10 & 10 \\
\hline
\end{tabular}

CIN, chemotherapy-induced neutropenia; ANC, absolute neutrophil count; DTI, direct-to-implant; ADM, acellular dermal matrix; POD, postoperative day; AC, doxorubicin, cyclophosphamide; TC, docetaxel, cyclophosphamide; AT, doxorubicin, docetaxel; FC, 5-fluorouracil, cyclophosphamide; FEC, 5-fluorouracil, cyclophosphamide, pharmorubicin. age of all the women was 46.89 in CIN group and 45.42 in non-CIN group, respectively. The CIN group had a mean body mass index of $23.57 \pm 4.5 \mathrm{~kg} / \mathrm{m}^{2}$, ranging from 18 to $30 \mathrm{~kg} / \mathrm{m}^{2}$. In total patients, 16 patients had hypertension and 22 patients had diabetes. None of the patients were active smokers in both CIN and non-CIN group. In the non-CIN group, chemotherapy was performed an average of 33.04 days after breast reconstruction, and in the CIN group, it took an average of 17.66 days. Drainage removal took 16.79 days in the non-CIN group and 15.81 days in the CIN group. The cancer stages in all the patients ranged from stage 0 to IV and total 74 patients were stage II (66.7\%). The mean value of lowest ANC in CIN group was 268.04 cells $/ \mu \mathrm{L}$.

Major infection requiring surgical management occurred in 4 patients $(7.1 \%)$ in the CIN group and $2(3.6 \%)$ in the non-CIN group (Table 2, Figure 1). Minor infection requiring antibiotics treatment occurred in 1 patient $(1.8 \%)$ in the both group (Figure 2). Capsular contracture occurred in $1(1.8 \%)$ in the CIN group and $4(7.3 \%)$ in the non-CIN group. Implant exposure requiring removal surgery occurred only in non-CIN group with 1 patient $(1.8 \%)$. Skin necrosis occurred in 7 patients $(12.5 \%)$ in CIN group and 11 patients (19.6\%) in non-CIN group. All complications did not show significant differences between the two groups.

\section{Discussion}

CIN is defined as an ANC per microliter of blood by inhibition of bone marrow precursors $(4,7)$. The generally accepted reference range for ANC in adults is 1,500 to 8,000 cells $/ \mu \mathrm{L}$. Fontanella et al. reported that $37 \%$ of the breast cancer patients experienced an ANC lower than 500 cells $/ \mathrm{mm}^{3}$ over the first four cycles of chemotherapy, and approximately $70 \%$ of the initial episodes occurred in first cycle (8). CIN cause severe infection, delay on chemotherapy, prolonged hospitalization, and even death. Common sites of infection in neutropenic patients include respiratory tract (35-40\%), bloodstream (15-35\%), urinary tract $(5-15 \%)$, skin and skin structure $(5-10 \%)$, gastrointestinal tract (5-10\%) (9). Staphylococcus species is the most common source of cutaneous origin, as well as Bacillus spp. and Corynebacterium spp. Age (over 65), female sex, malnutrition, lower baseline blood cell counts, poor performance status and reduced comorbidities were considered as risk factor of CIN (10).

Infections in implant based breast reconstruction with 
Table 2 Comparison of incidence of complication according to the CIN occurrence

\begin{tabular}{lccc}
\hline Complication & Non-CIN group, $\mathrm{n}(\%)$ & CIN group, n (\%) & P value \\
\hline Major infection & $2(3.6)$ & $4(7.1)$ & 0.679 \\
Minor infection & $1(1.8)$ & $1(1.8)$ & 1.000 \\
Capsular contracture & $4(7.3)$ & $1(1.8)$ & 0.206 \\
Implant rupture & $1(1.8)$ & 0 & 0.495 \\
Skin necrosis & $11(19.6)$ & $7(12.5)$ & 0.314 \\
\hline
\end{tabular}

CIN, chemotherapy-induced neutropenia.
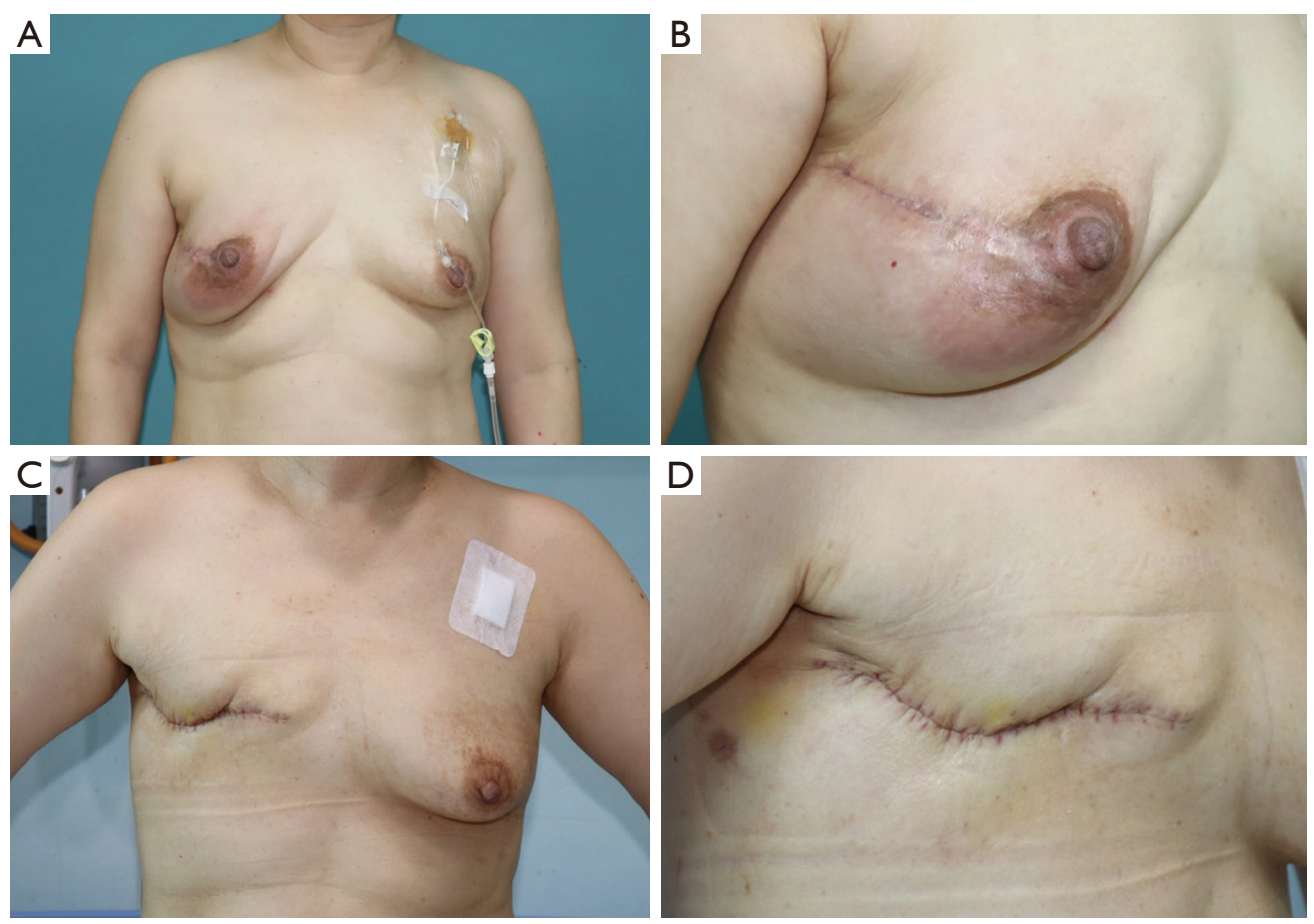

Figure 1 Clinical photograph of patient with major infection during the adjuvant chemotherapy. Fifty-three years old female patient started adjuvant chemotherapy (docetaxel, cyclophosphamide), 7 days after right breast reconstruction. (A,B) At the end of the third cycle, patient had breast infection with heatness, erythema and mild pain on her right breast. ANC was 228.90 cells/ $\mu \mathrm{L}, 2$ weeks before symptom started. The patient received intravenous antibiotics, but the symptom didn't resolved and seroma collection was identified by ultrasonography. (C,D) The patient got breast implant removed and infection was resolved. ANC, absolute neutrophil count.

implant were reported in $1 \%$ to $35 \%$ (11). Symptoms of infection include breast pain, swelling, erythema, fluid collection and may include fever. The main cause of immediate postoperative infection is intraoperative infection. Late infection is less common, however, can occur without obvious symptoms. There are many risk factors of infection, such as body mass index, diabetes, smoking, postmenopausal status, steroid therapy, lymph node resection, chemotherapy, chest wall radiation, and $\mathrm{ADM}$ used in the procedure. As infected condition persists, fibroblast growth is promoted, causing capsular contracture (12).

If there is no improvement with antibiotic treatment, surgical implant removal is required. When skin necrosis is accompanied, breast reduction is inevitable due to removal of necrotic skin, resulting in asymmetric breast. Delayed breast reconstruction with tissue expanders or autologous tissue can be next option, but it can be a great burden on 

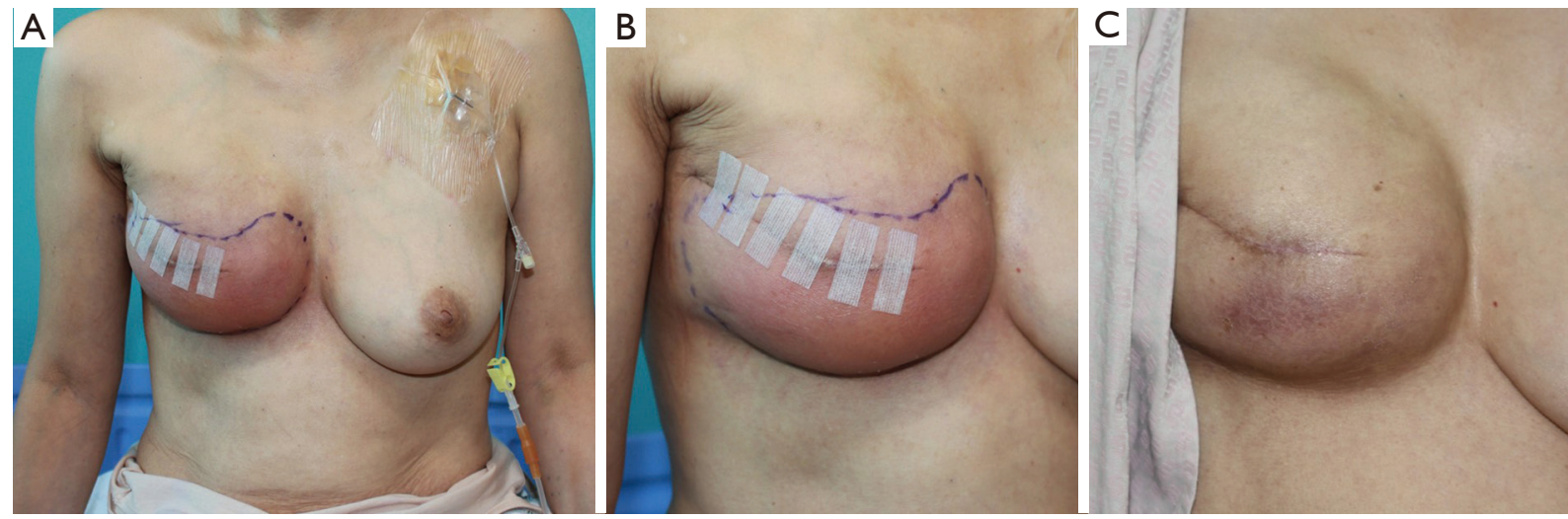

Figure 2 Clinical photograph of patient with minor infection during the adjuvant chemotherapy. Sixty-two years old female patient started adjuvant chemotherapy (doxorubicin, cyclophosphamide), 11 days after right breast reconstruction. (A,B) The patient had breast infection with severe erythema and heatness on her right breast at the end of the first cycle. ANC was 301.76 cells/ $\mu \mathrm{L}, 1$ week before the symptom started. (C) The patient received intravenous antibiotics and infection got improved after 1 week. ANC, absolute neutrophil count.

the patient, financially, psychologically, and physically. Therefore, inhibition of the immune system due to CIN in breast reconstruction patients using implants is a serious situation for both patients and doctors.

In this study, there were four cases of major infection in the CIN group, but there was no significant difference in the incidence of major and minor infections. The reason for the low incidence is that the patient was able to receive $\mathrm{IV}$ antibiotic treatment early by detecting CIN with regular blood tests during chemotherapy even if the patient had no symptoms. In addition, only complications that occurred between 1 month before and after the diagnosis of CIN were included. Therefore, complications that occurred more than 2 months after CIN occurrence were omitted, and the incidence of complications would be underestimated. To prevent the major infection, IV antibiotics were administered along with G-CSF in patients with febrile neutropenia. Initially, tazime and ornidazole were administered intravenous to prevent neutropenic sepsis, but vancomycin and tazime were administered together if breast symptoms such as heatness, erythema, and pain, exist. If symptoms persisted or worsened, the surgical implant removal is required. Since there was no significant difference in infection between the CIN group and the non-CIN group, based on the current policy, it would not be necessary to administer vancomycin from the beginning or immediately remove the implant when CIN occurs. Instead, close observation is necessary for breast infection, so hospitalization for 1 week is recommended. Moreover, patients should be aware that infection may occur several months after breast operation while continuing chemotherapy cycles.

Skin necrosis was shown in both group at $19.6 \%$ and $12.5 \%$, respectively. One of the important things in breast reconstruction is the blood circulation of the breast skin flaps. In the case of immediate skin flap necrosis after surgery, perforator injury of the skin flap after mastectomy can be major reason. Also, even if the same volume is mastectomy, blood perfusion becomes difficult if the patient's skin is thin. In case of skin necrosis, surgical revision is important because it can lead to implant exposure and infection due to skin necrosis. It has been reported that vasoconstriction and decreased palate activation can be induced due to chemotherapy-associated vascular toxicity (13). Additionally, chemotherapy impairs endothelial function, induce vascular and renal damage, oxidative stress, and thrombosis (13). Based on this, it should be noted that delayed skin necrosis can occur even in patients undergoing adjuvant chemotherapy. In further study, it is necessary to study flap circulation through routine transcutaneous $\mathrm{PO}_{2}$ monitoring when chemotherapy is performed.

In the CIN group, chemotherapy was started on average 17.66 days after breast reconstruction. Patients who showed wound problem, such as mild skin necrosis, began chemotherapy after the wound recovered. Chemotherapy is important because it improves patient survival rate. Although the impact of delaying chemotherapy is debatable, there is concern that it may, in the long term, have an adverse effect on disease free and overall survival $(14,15)$. The time required for chemotherapy was 41 days for patients with breast reconstruction and 53 days for patients without breast 
reconstruction (16). In general, it takes only a few weeks to treat the infection of breast reconstruction (17) and it does not differ greatly in the treatment of skin necrosis. Therefore, delay in chemotherapy can be considered in patients with wound problems.

In the 1980s, cyclophosphamide, methotrexate, and 5-fluorouracil (CMF) were used as standard chemotherapy agents. Later, several drugs such as doxorubicin, epirubicin, docetaxel, and paclitaxel were added through the clinical trial process. Today, different regimen have been chosen for standard treatment for each patient according to cancer size, grade, lymph node status, hormone receptor status, HER2 status and genetic profile $(18,19)$. Several studies showed different CIN incidence according to the different chemotherapy regimen. Boér et al. reported a higher febrile neutropenia in the TAC (docetaxel, doxorubicin, cyclophosphamide) group (76\%) than in the FAC (5-fluorouracil, doxorubicin, cyclophosphamide) group (22\%) (20). Leonard et al. ed reported 29\% of the CMFbased regimens group had a neutropenic event, and $28 \%$ of the anthracycline-based regimen group had a difference in neutropenic event occurrence pattern by cycle in the two groups (21). In this study, of a total of 111 patients, 74 patients were stage II and 75 patients received AC regimen. There were no statistically differences in the adjuvant chemotherapy regime between two groups. Therefore, we believe that different chemotherapy regimens have little effect on the relationship between CIN and breast complication in this study.

There are some limitations. It is difficult to confirm a clear causality between CIN and breast complication base on the time of the patient's hospital visit, the time when the blood test was performed, and the time when breast complication was first found. Therefore, there is a selection bias including only complication that occurred 1 month before and after CIN occurrence. However, as far as the author knows, it is the first study that analyzed the relationship between CIN and breast implant-associated complications during the adjuvant chemotherapy that anyone may be curious about, and it will be useful to solves this question.

\section{Conclusions}

CIN do not significantly increase the incidence of severe complications in the patients who received adjuvant chemotherapy after DTI immediate breast reconstruction. However, starting adjuvant chemotherapy should be carefully considered in patients with wound problems, and close observation is required for CIN patients, and may need aggressive intravenous antibiotic management or surgical implant removal. It is also recommended for surgeon and patients to aware late complication can be occur during the chemotherapy cycles.

\section{Acknowledgments}

Funding: None.

\section{Footnote}

Reporting Checklist: The authors have completed the STROBE reporting checklist. Available at http://dx.doi. org/10.21037/apm-21-508

Data Sharing Statement: Available at http://dx.doi. org/10.21037/apm-21-508

Peer Review File: Available at http://dx.doi.org/10.21037/ apm-21-508

Conflicts of Interest: All authors have completed the ICMJE uniform disclosure form (available at http://dx.doi. org/10.21037/apm-21-508). The authors have no conflicts of interest to declare.

Ethical Statement: The authors are accountable for all aspects of the work in ensuring that questions related to the accuracy or integrity of any part of the work are appropriately investigated and resolved. The study was conducted in accordance with the Declaration of Helsinki (as revised in 2013). The study was approved by institutional review board of Yeungnam University Hospital (No. 202101-043: the registration number of ethics board) and informed consent were taken from all the patients of clinical photographs at the figure.

Open Access Statement: This is an Open Access article distributed in accordance with the Creative Commons Attribution-NonCommercial-NoDerivs 4.0 International License (CC BY-NC-ND 4.0), which permits the noncommercial replication and distribution of the article with the strict proviso that no changes or edits are made and the original work is properly cited (including links to both the formal publication through the relevant DOI and the license). See: https://creativecommons.org/licenses/by-nc$\mathrm{nd} / 4.0 \%$. 


\section{References}

1. of chemotherapy on postoperative complications after mastectomy and immediate breast reconstruction. Arch Surg 2010;145:880-5.

2. Early Breast Cancer Trialists' Collaborative Group (EBCTCG). Effects of chemotherapy and hormonal therapy for early breast cancer on recurrence and 15year survival: an overview of the randomised trials. Lancet 2005;365:1687-717.

3. Coleman RE. Current and future status of adjuvant therapy for breast cancer. Cancer 2003;97:880-6.

4. Wilson-Royalty M, Lawless G, Palmer C, et al. Predictors for chemotherapy-related severe or febrile neutropenia: a review of the clinical literature. J Oncol Pharm Pract 2001;7:141-7.

5. Crawford J, Dale DC, Lyman GH. Chemotherapyinduced neutropenia: risks, consequences, and new directions for its management. Cancer 2004;100:228-37. Erratum in: Cancer 2004;100:1993-4.

6. Yule GJ, Concannon MJ, Croll G, et al. Is there liability with chemotherapy following immediate breast construction? Plast Reconstr Surg 1996;97:969-73.

7. Bhatt V, Saleem A. Review: Drug-induced neutropenia-pathophysiology, clinical features, and management. Ann Clin Lab Sci 2004;34:131-7.

8. Fontanella C, Bolzonello S, Lederer B, et al. Management of breast cancer patients with chemotherapy-induced neutropenia or febrile neutropenia. Breast Care (Basel) 2014;9:239-45.

9. Nesher L, Rolston KV. The current spectrum of infection in cancer patients with chemotherapy related neutropenia. Infection 2014;42:5-13.

10. Lyman GH, Lyman CH, Agboola O. Risk models for predicting chemotherapy-induced neutropenia. Oncologist 2005;10:427-37.

Cite this article as: Sakong Y, Choi MK, Lee JH. The impact of chemotherapy-induced neutropenia on the outcome of direct-to-implant immediate breast reconstruction. Ann Palliat Med 2021;10(5):5181-5187. doi: 10.21037/apm-21-508
11. Washer LL, Gutowski K. Breast implant infections. Infect Dis Clin North Am 2012;26:111-25.

12. Adams WP Jr. Capsular contracture: what is it? What causes it? How can it be prevented and managed? Clin Plast Surg 2009;36:119-26, vii.

13. Cameron AC, Touyz RM, Lang NN. Vascular complications of cancer chemotherapy. Can J Cardiol 2016;32:852-62.

14. Styblo TM, Lewis MM, Carlson GW, et al. Immediate breast reconstruction for stage III breast cancer using transverse rectus abdominis musculocutaneous (TRAM) flap. Ann Surg Oncol 1996;3:375-80.

15. Johnson A. The timing of treatment in breast cancer: gaps and delays in treatment can be harmful. Breast Cancer Res Treat 2000;60:201-9.

16. Allweis TM, Boisvert ME, Otero SE, et al. Immediate reconstruction after mastectomy for breast cancer does not prolong the time to starting adjuvant chemotherapy. Am J Surg 2002;183:218-21.

17. Macadam SA, Mehling BM, Fanning A, et al. Nontuberculous mycobacterial breast implant infections. Plast Reconstr Surg 2007;119:337-44.

18. Rampurwala MM, Rocque GB, Burkard ME. Update on adjuvant chemotherapy for early breast cancer. Breast Cancer (Auckl) 2014;8:125-33.

19. Gradishar WJ, Anderson BO, Balassanian R, et al. NCCN guidelines insights: breast cancer, version 1.2017. J Natl Compr Canc Netw 2017;15:433-51.

20. Boér K, Juhos É, Pintér T, et al. Adjuvant therapy of breast cancer with docetaxel-containing combination (TAC)a Hungarian experience in the BCIRG 001 trial. Pathol Oncol Res 2003;9:166-9.

21. Leonard RC, Miles D, Thomas R, et al. Impact of neutropenia on delivering planned adjuvant chemotherapy: UK audit of primary breast cancer patients. Br J Cancer 2003;89:2062-8. 